Proceedings of Anticancer Research

Research Article

\title{
Effect of Periodontal Serial Therapy Combined with Orthodontics on Periodontal Parameters in Patients with Periodontitis
}

Lu Bai

Department of Stomatology, Affiliated Hospital of Chifeng University, Chifeng 024000, Inner Mongolia, China

[Abstract] Objective: To investigate the clinical efficacy of periodontal sequence in combination with orthodontic treatment of patients with periodontitis. Methods: Retrospective analysis of periodontitis patients (54 cases) treated at our hospital between February 2018 and June 2019. The clinical data of all the enrolled participants were subjected to periodontal serial combined orthodontic treatment, and all the registered participants were observed at the initial consultation, periodontal serial treatment1 Periodontal parameters at the end of months and at the end of orthodontic treatment, serial periodontal treatment and orthodontic treatment for all enrolled individuals, x-rays and Oral examinations were performed, and the results were analyzed. Results: Compared to the initial visit, all enrolled individuals had a higher plaque index (PLI) after 1 month of periodontal treatment and at the end of orthodontic treatment. Bleeding index (BI), periodontal probe depth (PD), and periodontal attachment loss (CAL) levels were all lower than those detected at the initial visit. The difference was statistically significant $(\mathrm{P}<0.05)$; after serial periodontal treatment and orthodontic treatment, the $\mathrm{x}$-ray results showed that the patients' Alveolar bone resorption or regeneration ceased; The results of the oral examination showed that the patient's occlusal relationship improved significantly and basically returned to normal. Status, a masticatory function was significantly improved, and individual tooth loosening due to occlusal trauma was improved considerably. Conclusion: The combination of periodontal sequence and orthodontic treatment of patients with periodontitis is beneficial to enhance periodontal parameters, improve occlusal relationships, and improve masticatory function.

Keywords: Periodontitis; Periodontal sequence; Orthodontics; Periodontal indicators
Publication date: September, 2020

Publication online: 30 September, 2020

*Corresponding author: Lu Bai,11118227@163.com

Periodontitis is a chronic inflammation caused by bacteria in plaque that invades the periodontal tissues. The main clinical manifestations are resorption, which can easily cause damage to the gingiva, periodontal membrane, alveolar bone and other periodontal supporting tissues, and will seriously endanger patients - oral health ${ }^{[1-2]}$. For patients with periodontitis requiring orthodontic treatment, the loss of attachment limits the patient's ability to tolerate orthodontic pressure, resulting in Clinical medicine is more difficult ${ }^{[3]}$. To improve this situation, the present study used periodontal sequences in combination with orthodontics to treat patients with periodontitis, to further observe its effect on the patients' periodontal the impact of the indicator. This is illustrated below.

\section{Information and methodology}

\subsection{General information}

Review of periodontitis treated at our hospital between February 2018 and June 2019 Clinical data of the patients (54), 24 males and 30 females; age 36-48 years, mean age $(41.87 \pm 2.72)$ years; disease duration $4-16$ months, mean duration of disease $(9.75 \pm 2.31)$ months; disease Grading: 32 cases of mild, 22 cases of moderate.

\subsection{Inclusion criteria and Exclusion criteria}

(1) Inclusion criteria: Those with probing bleeding, gingival inflammation, probing attachment loss less than 
$5 \mathrm{~mm}$. Periodontal probing depth of less than $6 \mathrm{~mm}$; $\mathrm{X}$-ray examination showed that there was horizontal or angular absorption in the alveolar bone, and the absorption was less than half of the root length; Clinical data and imaging data are complete.

(2) Exclusion criteria: Recent use of antibiotics; Coagulation dysfunction; Combined metabolic system and immune system diseases; Persons with severe functional impairment of heart, liver, kidney and other organs; Persons with expression disorders or psychiatric disorders.

\subsection{Methodology}

The treatment plan is determined at the first visit, and oral hygiene education is provided to patients to promote awareness of the daily patients are taught the importance of oral hygiene maintenance, the proper way to brush their teeth, and flossing to clean between teeth. This is followed by a periodontal sequence of treatment by performing a full mouth supragingival scaling, disposable subgingival scraping and root planing on the patient. At the same time give anti-infection drug treatment, the treatment time: 1-3 months, during the treatment period and continually strengthen plaque control. One month after the end of the procedure, the patient's periodontal inflammation condition will be re-examined, and orthodontic treatment will be carried out for those who have good periodontal inflammation control condition. Patients with poor periodontal inflammation control will continue to be observed for 3 months to ensure the plaque control reaches the standard, and then orthodontic treatment will be carried out. Orthodontic treatment: first clean the plaque on the patient's teeth, and then set the angle of the straight filament arch according to the deformity of the patient's teeth. The orthodontic appliance is installed in the brackets of the affected teeth, and the straight wire arch appliance is adjusted appropriately to increase the corrective strength. After all the above operations are completed, the orthodontic appliances are fixed. The orthodontic time is 14-24 months, with follow-up appointments every 4-6 weeks and cleaning every 6 months.

\subsection{Evaluation indicators}

Periodontal probing at the initial consultation, 1 month after serial periodontal treatment, and at the end of orthodontic treatment (Williams scale) for all enrolled periodontal parameters, including plaque index (PLI): score 0-3 degrees, bleeding index (BI): bleeding was recorded through a periodontal probe gently inserted into the bottom of the pocket and $30 \mathrm{~s}$ after removal of the examination. Graded 0-5, periodontal probe depth (PD): distance from the gingival margin to the bottom of the pocket, periodontal attachment loss (CAL): through the probe. The extent of the periodontal pocket was examined, the location of the enamel bone boundary was explored when the probe was withdrawn, and the distance from the gingival margin to the bone boundary of the opposing enamel tooth was measured. Degree of attachment loss = pocket depth - distance from the gingival margin to the bone boundary of the opposing enamel teeth. After the serial periodontal treatment and orthodontic treatment, the oral conditions were examined by X-ray film.

\subsection{Statistical methods}

Data processing was performed using SPSS 24.0 software to represent the measures, and comparisons between multiple groups were made. One-way ANOVA was used, and $\mathrm{P}<0.05$ was considered a statistically significant difference.

\section{Results}

\subsection{Periodontal Indicators}

Compared to the initial consultation, at 1 month after periodontal treatment and at the end of orthodontic treatment for all enrolled individuals, the PLI, the BI, $\mathrm{PD}$, and CAL levels were all lower than those tested at the initial visit. The difference was statistically significant $(\mathrm{P}<0.05)$. See Table 1.

Table 1. Comparison of periodontal indicators across time for all enrolled participants $(x \pm s)$

\begin{tabular}{ccccc}
\hline Groups & PLI & BI & PD $(\mathrm{mm})$ & CAL $(\mathrm{mm})$ \\
\hline $\begin{array}{c}\text { At first consultation }(\mathrm{n}=54) \\
1 \text { month after periodontal treatment } \\
(\mathrm{n}=54)\end{array}$ & $2.21 \pm 0.84$ & $2.53 \pm 0.51$ & $4.18 \pm 1.05$ & $3.04 \pm 1.53$ \\
$\begin{array}{c}\text { At the end of orthodontic treatment } \\
(\mathrm{n}=54)\end{array}$ & $0.86 \pm 0.43 \mathrm{a}$ & $0.97 \pm 0.32 \mathrm{a}$ & $2.35 \pm 0.78 \mathrm{a}$ & $1.94 \pm 0.78 \mathrm{a}$ \\
$F$ & $1.45 \pm 0.64 \mathrm{a}$ & $1.13 \pm 0.38 \mathrm{a}$ & $2.14 \pm 0.82 \mathrm{a}$ & $2.03 \pm 0.65 \mathrm{a}$ \\
$P$ & 49.522 & 235.388 & 85.585 & 17.923 \\
\hline
\end{tabular}

Note: ${ }^{a} P<0.05$ compared to the initial consultation. 


\subsection{X-rays and oral examination findings}

After periodontal serial therapy and orthodontic treatment, the results of X-rays showed that the patient Alveolar bone resorption or regeneration ceased; the results of the oral examination showed that the patient's occlusal relationship improved significantly and basically returned to normal. Condition, chewing function is significantly improved, and individual tooth looseness due to occlusal trauma is enhanced considerably.

\section{Discussion}

Many previous researchers have concluded that periodontitis patients have less periodontal tissue support and that orthodontic treatment of patients with this condition is comfortable on the periodontal tissue. Damage, so orthodontic treatment is not advocated for patients with periodontitis ${ }^{[4]}$. In recent years, more and more studies have demonstrated that orthodontic treatment given to patients with well-controlled periodontal inflammation does not destroy periodontal tissue. Instead, useful improvement in periodontal status can be achieved ${ }^{[5-6]}$. Occlusal trauma is exacerbated in patients with periodontitis when combined with occlusal trauma, especially in the presence of significant alveolar bone resorption and tooth loosening. Periodontal tissue damage, therefore, orthodontic treatment of patients with periodontitis requires the removal of occlusal interference and occlusal trauma to promote the affected tooth Fixation and periodontal tissue healing have a positive impact on reducing periodontal tissue damage.

In general, during orthodontic treatment, the teeth are moved by force, and a small amount of resorption of the alveolar bone can occur, especially in periodontitis gastroscopy. Treatment with orthodontic procedures can result in significant and rapid alveolar bone resorption ${ }^{[7]}$. Therefore, orthodontic treatment of patients with periodontitis must be performed to control the inflammatory condition of the periodontium and patients are instructed to develop good Oral hygiene habits and regular (3-6 months/visit) periodontal review and implementation of appropriate periodontal treatment based on the patient's periodontal status to Stabilize the patient's periodontal function. The orthodontic treatment can also eliminate the oral plaque accumulation area and promote rapid recovery of periodontal tissues ${ }^{[8]}$. $\mathrm{Xu}$ Feihu et al ${ }^{[9]}$ showed that periodontitis patients with orthodontic treatment with periodontal sequence therapy, which is beneficial to improve clinical treatment Efficiency, improve patient PLI, BI, PD, CAL levels.PLI, BI, PD, CAL are clinical assessments. It is an essential index of oral hygiene and the effect of periodontal disease prevention and treatment, in which PLI can reflect the degree of posterior plaque, the higher the degree of the plate on the surface of the teeth. The thicker the thickness; BI mainly refers to the use of a blunt probe to gently probe the periodontal pocket, remove the probe for $30 \mathrm{~s}$ bleeding, which can adequately reflect gingival inflammation. The higher the grade, the more severe the inflammation; PD is an essential indicator for diagnosing periodontitis and accurately measures the depth of the periodontal pocket; CAL can Reflecting the destruction of periodontal support tissues, this study used a combination of periodontal sequences and orthodontics to treat patients with periodontitis, and the results showed that, compared to the primary PLI, BI, PD, and CAL levels at the end of orthodontic treatment were higher in all enrolled participants at 1 month after periodontal treatment and at the end of orthodontic treatment compared to the time of surgery. Lower than the initial test results, suggesting that this treatment is effective in improving the patient's periodontal parameters. During the periodontal sequence, patients were treated with full supragingival scaling, disposable subgingival scraping, and root surface smoothing, and the patient's teeth were Surface plaque, calculus, pockets of inflammatory tissue, infected bone, etc can be effectively removed, and then the local inflammation of the patient's oral cavity. Control and anti-infective medication were given to further remove deep subgingival plaque from the oral cavity and lay the foundation for subsequent orthodontic treatment. The results of this study showed that after serial periodontal treatment and orthodontic treatment, the patient's alveolar bone resorption or regeneration was demonstrated by X-ray examination results. The effects of oral examination showed that the patient's bite relationship improved significantly, basically returned to a healthy state, and chewing function was enhanced considerably. The individual teeth loosening due to occlusal trauma is enhanced considerably. The reason for this is that after orthodontic treatment is completed, the patient's teeth are aligned, and plaque removal can be achieved, and the appearance of plaque can be effectively treated - control, which is more conducive to periodontal tissue recovery ${ }^{[10]}$.

In summary, the combination of periodontal sequence and orthodontic treatment of patients with periodontitis is beneficial for improving periodontal parameters, improving occlusal relationships, enhancing masticatory function, and contributing to patient benefit.

\section{References}


[1] Ye ZF, Zhu XQ, Fang D. Changes in serum levels of CRP IL-8 and TNF- $\alpha$ in periodontal treatment of chronic periodontitis with coronary heart disease[J]. Zhejiang Clin Med, 2019, 21(2): 167-168.

[2] Yang N. Effect of different antibiotic-assisted periodontal essential therapy on the efficacy of invasive periodontitis[J]. Chinese Drugs and Clinical, 2019, 19(2): 278-279.

[3] Guo SQ. Observation of the clinical effect of orthodontic combined with essential periodontal therapy applied to the treatment of periodontal disease[J]. Shanxi Medical Journal, 2019, 48(11): 1307-1309.

[4] Tang CM, Zhao HJ, Xu G, et al. Effects of periodontal tissue regeneration combined with orthodontics on serum inflammatory factor levels in periodontitis patients and investigation of its efficacy[J]. Modern Biomedical Progress, 2017, 17(20): 39653968.

[5] Yang XR, Li JK, Zhang JJ, et al. The efficacy of combined periodontal and orthodontic treatment of patients with invasive periodontitis and its impact on periodontal function[J]. Chinese Journal of Gerontology, 2017, 37(11):2769-2770.

[6] Huang Z, Shi J, Chen G, et al. A case of five-year follow-up of periodontal-orthodontic-implant-prosthetic combination treatment of severe chronic periodontitis $[\mathrm{J}]$. Chinese Journal of Stomatology, 2017, 52(2): 81-85.

[7] Zhou WJ, Che YL, Su Y, et al. The effect of periodontal tissue regeneration combined with orthodontic treatment on periodontal status and satisfaction in patients with periodontitis[J]. Progress in Modern Biomedicine, 2019, 19(9): 1683-1686.

[8] Miao ZX, Wang JB, Fang B. Effect of orthodontic micro-implants on periodontal inflammation and MMP-2 and MMP-9 in patients with Anse II malocclusion[J]. Hainan Medicine, 2019, 30(12): 1578-1580.

[9] Xu FH, Shen X. Analysis of the effect of the periodontal sequence in the orthodontic process of patients with periodontitis[J]. Journal of Xuzhou Medical College, 2017, 37(12): 860862.

[10] Li WB, Zhang Q, Li ZJ. Efficacy of periodontal combined orthodontic treatment of patients with invasive periodontitis and its effect on the clinical periodontal index and periodontal function[J]. Journal of Clinical and Experimental Medicine, 2019, 18(10): 1113-1116. 